\title{
Standard pleural interventions are not high-risk aerosol generating procedures
}

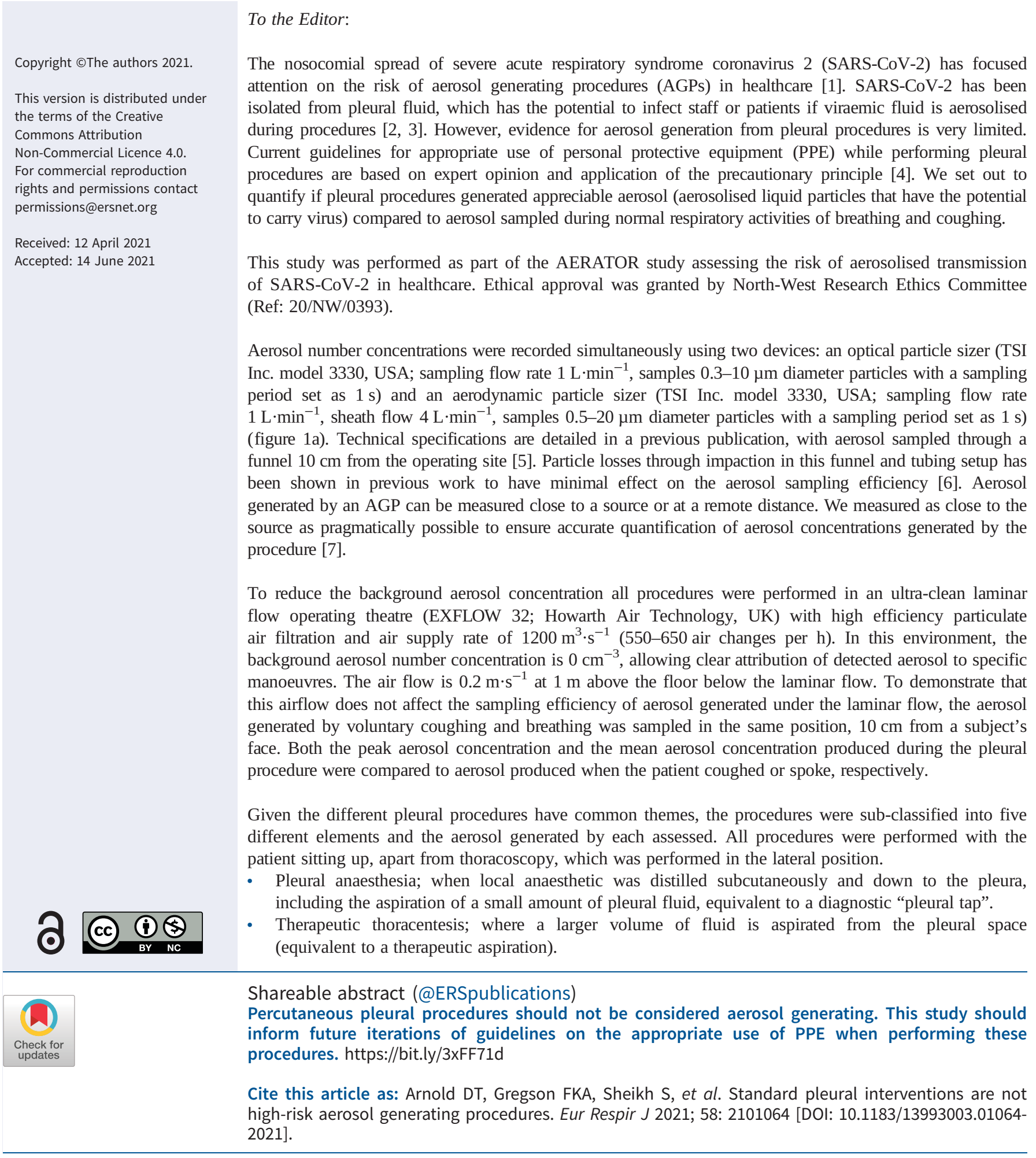



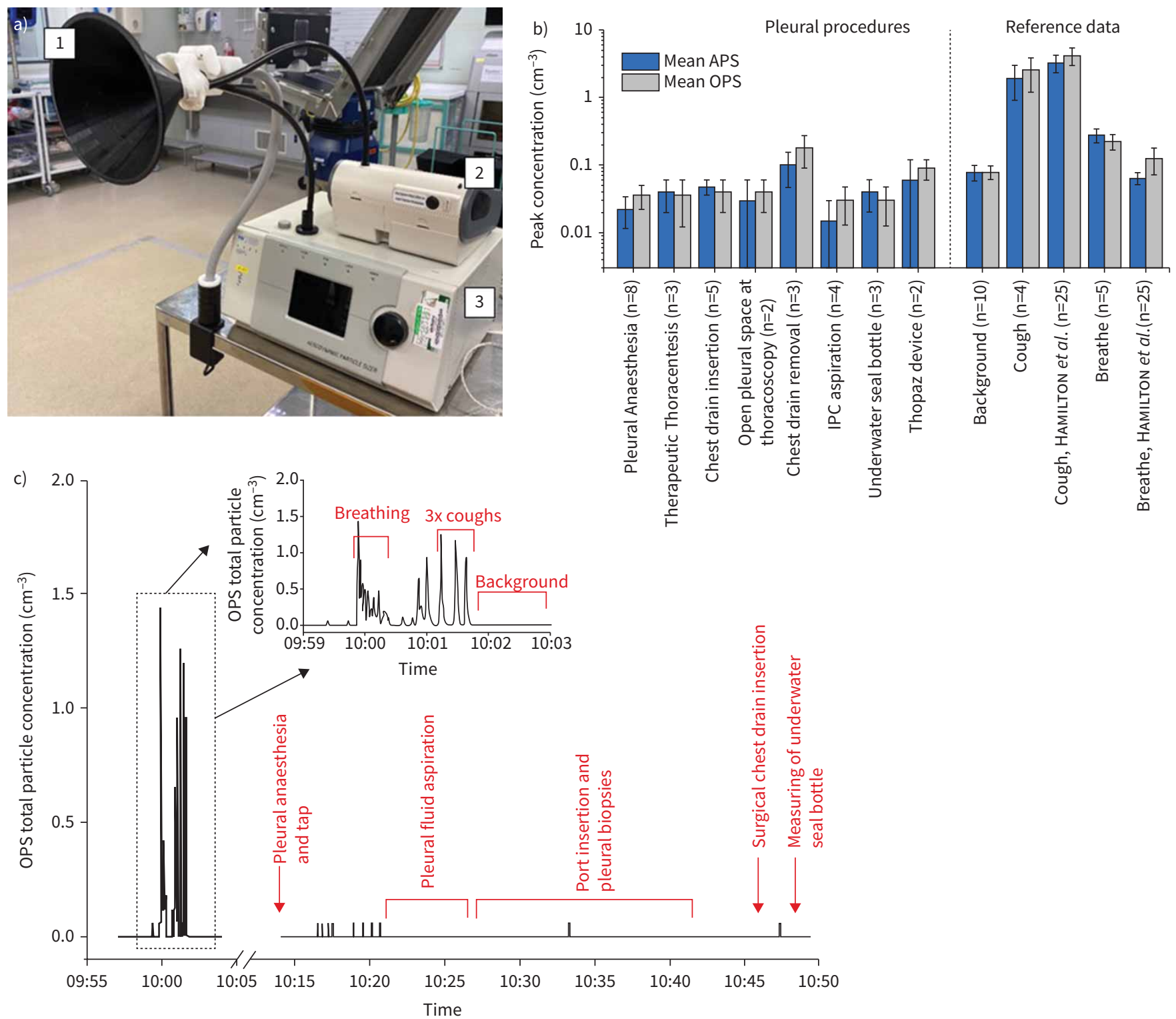

FIGURE 1 a) Sampling equipment set-up. 1) Sampling funnel; 2) optical particle sizer (OPS); 3) aerodynamic particle sizer (APS). b) Bar chart (log-scale) showing the peak aerosol number concentration sampled by the APS and OPS methods during different procedural elements compared to mean aerosol number concentrations measured during breathing and peak aerosol number concentrations measured during cough. c) Particle concentration over time (linear scale) during a single thoracoscopy compared to aerosol production from the patient breathing and coughing pre-procedure. Pre-procedure aerosol concentrations are compared to that from healthy subjects in HAMILToN et al. [5]. Error bars represent SEM. IPC: indwelling pleural catheter.

- Chest drain insertion; including indwelling pleural catheter (IPC) and surgical chest drain insertion.

- Open pleural space procedures; during thoracoscopy with a port in situ allowing the movement of air between the pleural space and the atmosphere.

- Chest drain removal.

We also measured any aerosol produced from fluid management systems of:

- Underwater seal chest drain bottle (Rocket Medical)

- IPC bottle aspiration (BD)

- $\quad$ Thopaz+ Digital Chest Drainage System (Medela)

10 patients (who were SARS-CoV-2 PCR negative) requiring pleural procedures (three medical thoracoscopies, three IPC insertions (15.5 Fr), one therapeutic aspiration (6 Fr), three IPC removals)) were recruited to the study, with two further patients with chest tubes already in situ for pneumothorax with ongoing air leak. The majority of patients were male (10/12) with a median age of 76 (interquartile range 72-79) years. 
Figure 1b (logarithmic $y$-axis) shows the peak aerosol number concentration sampled during each procedure compared to peak number concentrations from coughing and mean number concentrations from breathing. For most procedures, the peak number concentration was of similar magnitude to or less than the mean aerosol number concentration measured during breathing from the same patients or from healthy volunteers from a previous study, and was significantly less than the peak number concentration detected from a cough [5]. The mean concentration for all procedures is typically much less (up to two orders of magnitude) smaller than the mean concentration sampled when a subject is breathing. Again, it should be stressed that breathing is a sustained activity while coughs and these clinical interventions lead to transient events.

Figure 1c illustrates the difference between the particle number concentration sampled during a single procedure compared to breathing or coughing for a patient undergoing a medical thoracoscopy.

This study shows that percutaneous instrumentation of the pleura does not result in significant aerosol generation. Total aerosol generation during these procedures was significantly below the number concentration produced by breathing or coughing.

Current British Thoracic Society guidelines recommend that "closed pleural procedures such as pleural aspirations and chest drain insertion can be undertaken in level 1 PPE (surgical mask and visor, as well as gown and gloves)" whereas "open procedures such as thoracoscopy and IPC insertion, where pleural fluid may splash, should still be considered AGP [4]. Therefore, level 2 PPE should be worn (FFP3 mask, long sleeved gown, gloves, eye protection)". On the basis of this evidence, pleural procedures are not aerosol generating and additional PPE (above that indicated for routine patient care) is not required, although eye protection should be worn given the risk of splash.

It is well recognised that pleural procedures, especially those that generate a negative intrathoracic pressure (e.g. therapeutic thoracentesis) can induce a cough in participants. We would therefore recommend the patient be asked to wear a surgical facemask, which has been shown to significantly reduce aerosol produced during cough [5].

Pleural fluid management systems such as underwater seal chest tube bottles have also been seen as a source of aerosol generation, with several studies advocating the use of antiviral filters. DuFFy et al. [8] assessed aerosol generation by bubbling air at different rates through an underwater seal bottle, sampling a maximum aerosol concentration of particles (within the same size range to those studied here, $0.3-10 \mu \mathrm{m}$ ) during the bubbling process of $\sim 4100 \mathrm{ft}^{-3}$, caused by atomisation of the water. This equates to a peak number concentration of $\sim 0.14 \mathrm{~cm}^{-3}$, which is similar to the peak concentrations that we observed during the fluctuations of sampled aerosol number concentration during pleural procedures. We show that the peak number concentration sampled during the pleural procedures was similar to that sampled during the background measurement, orders of magnitude smaller than that sampled during a cough and was never greater than the mean number concentration sampled during a period of quiet breathing. However, given our sample size for underwater seal bottles is small $(n=3)$ and the mitigating factors are simple, we feel guidance should still encourage the use of viral filters or Thopaz devices until further evidence is gathered, especially in pneumothoraces with high air leaks.

In summary, using two methodologies to measure aerosol emission with no background aerosol interference, this study has shown that percutaneous pleural procedures are non-aerosol generating. We hope this will inform future iterations of guidelines on the appropriate use of PPE when performing these procedures.

David T. Arnold ${ }^{1}$, Florence K.A. Gregson ${ }^{2}$, Sadiyah Sheikh ${ }^{2}$, Fergus W. Hamilton ${ }^{3,4}$, Hugh Welch ${ }^{1}$, Alexandra Dipper ${ }^{1}$, George W. Nava ${ }^{1}$, AERATOR group ${ }^{5}$, James W. Dodd $\oplus^{1,3}$, Amelia O. Clive ${ }^{1}$, Bryan R. Bzdek ${ }^{2}$, Jonathan P. Reid ${ }^{2}$ and Nick A. Maskell ${ }^{1}$

${ }^{1}$ Academic Respiratory Unit, University of Bristol, Bristol, UK. ${ }^{2}$ Bristol Aerosol Research Centre, School of Chemistry, University of Bristol, Bristol, UK. ${ }^{3} \mathrm{MRC}$ Integrative Epidemiology Unit, University of Bristol, Bristol, UK. ${ }^{4}$ Infection Sciences, North Bristol NHS Trust, Bristol, UK. ${ }^{5} \mathrm{~A}$ list of members of the AERATOR group can be found in the acknowledgements section. 
AERATOR group (in alphabetical order): The AERATOR group consists of (in alphabetical order): David T. Arnold, Jules Brown, Bryan R. Bzdek, Andrew Davidson, James W. Dodd, Mark Gormley, Florence K.A. Gregson, Fergus W. Hamilton; Nick A. Maskell, James Murray, Johannes Keller, Anthony E. Pickering, Jonathan P. Reid, Sadiyah Sheikh and Andrew Shrimpton.

Author contributions: D.T. Arnold, F.W. Hamilton and N.A. Maskell developed the study idea. F.K.A. Gregson, F.W. Hamilton, J.W. Dodd, B.R. Bzdek and J.P. Reid developed the sampling design. D.T. Arnold, S. Sheikh, F.W. Hamilton, H. Welch, A. Dipper, G.W. Nava and N.A. Maskell collected the primary aerosol data. F.K.A. Gregson, S. Sheikh, B.R. Bzdek and J.P. Reid analysed the aerosol data. All authors were involved in the writing of the manuscript.

Conflict of interest: None declared.

Support statement: The AERATOR study was funded by an NIHR/UKRI COVID-19 Rapid Rolling Call (grant number COV0333). D.T. Arnold is funded by an NIHR Doctoral Research Fellowship. F.W. Hamilton is funded by a GW4 Wellcome Trust Fellowship. B.R. Bzdek acknowledges support from the Natural Environment Research Council (NE/P018459/1). Funding information for this article has been deposited with the Crossref Funder Registry.

\section{References}

1 Richterman A, Meyerowitz EA, Cevik M. Hospital-acquired SARS-CoV-2 infection: lessons for public health. JAMA 2020; 324: 2155-2156.

2 Malik MI, Fox N, Chopra A, et al. Positive pleural fluid RT-PCR for virus detection in SARS-CoV-2 pneumonia. QJM 2020; 113: 888-889.

3 Mei F, Bonifazi M, Menzo S, et al. First detection of SARS-CoV-2 by real-time reverse transcriptase-polymerase chain reaction assay in pleural fluid. Chest 2020; 158: e143-e146.

4 Hallifax R, Wrightson J, Bibby A, et al. Pleural Services during the COVID-19 Pandemic. www.brit-thoracic.org. uk/document-library/quality-improvement/covid-19/pleural-services-during-covid-19-pandemic/ Date last accessed 11 March, 2021. Date last updated: 20 May, 2021.

5 Hamilton F, Gregson F, Arnold D, et al. Aerosol emission from the respiratory tract: an analysis of relative risks from oxygen delivery systems. medRxiv 2021; preprint [https://doi.org/10.1101/2021.01.29.21250552].

6 Gregson FKA, Watson NA, Orton CM, et al. Comparing aerosol concentrations and particle size distributions generated by singing, speaking and breathing. Aerosol Sci Technol 2021; 55: 681-691.

7 Walker JS, Archer J, Gregson FKA, et al. Accurate representations of the microphysical processes occurring during the transport of exhaled aerosols and droplets. ACS Cent Sci 2021; 7: 200-209.

8 Duffy C, Kidd A, Francis S, et al. Chest drain aerosol generation in COVID-19 and emission reduction using a simple anti-viral filter. BMJ Open Respir Res 2020; 7: e000710. 